\title{
The Coast Effect in the Variations of the Earth's Electromagnetic Field
}

\author{
By I.I. RokitJANSKY, \\ Institute of the Physics of the Earth, Moscow \\ P.K. SenKo, \\ Institute for Arctic and Antarctic Research, Leningrad \\ S.M. Mansurov, J.K. Kalinin and G.A. Fonarev \\ Institute of Terrestrial Magnetism, Ionosphere and Radio-wave Propagation, Moscow
}

\begin{abstract}
The coast effect $[\mathrm{CE}]$ means an influence of the boundary of two different by their electromagnetic characteristics, mediums-land and sea-on the alternative electromagnetic fields.

Here are mentioned the properties of the coast effect for a wide range of variation periods from millimicrosecond up to the tenths of the minutes.

The coast effect for the variations with the periods from the units of seconds to the tenths of minutes is divided in 2 types by the look of polarization of the primary field as to the coast $\mathrm{CE}-1$ and $\mathrm{CE}-2$.

Here are given experimental data by $\mathrm{CE}-1$ for the area of the stations Mirny (Antarctida) and CE-2 for the station Alushta (coast of the Black Sea).
\end{abstract}

The coast effect $[\mathrm{CE}]$ in geophysics appears as a complicated phenomenon, displaying in a wide range of periods of electromagnetic field occilations: from millimicroseconds up to the tenths of minutes. The sources of the electromagnetic field are usually above land or above sea. The primary fields varying in time as a result of interaction with the coast create the secondary fields, stipulating CE. In a theoretical consideration of a phenomenon it is natural to consider sea in a way of a wedge. However a strict decision of an electrodynamic task for a wedge-form area has not been found yet. From a number of approximate methods one should distinguish a method, connected with radiowave propagation across and along the coast. It consists of the application of Grin's theorem and impedance boundary conditions on the sea and land surfaces. Such a task is possible with $T<10^{-4}$ sec. The main teoretical and experimental results concerning $\mathrm{CE}$ for radiowaves are the following.

1) In a wave transition to a less-conductive area an accelerated speed of the amplitude decrease is observed.

2) In a wave transition to an area with larger conductivity a speed of an amplitude 
decrease becomes lower; under some conditions an amplitude increase takes place with the removal from the source (recovery effect).

3) If a source and an observation point are on one and the same side of the coast line, then in transition in the normal direction to the coast line there is observed a small occilation of an amplitude and a phase. If a source and an observation point are on different sides, then an aperiodical decrease of disturbance takes place.

4) There is observed a declination of radiowave propagation direction from a rectilinear direction (the coast refraction).

The investigation results on $\mathrm{CE}$ of radiowaves are summarized in [1]. The usage of the above-mentioned method for long-periodical variations ( $T$-the units of seconds-the tenths of minutes) cannot go with a successively controlled accuracy due to a slowness of running processes. Therefore a particularly important role is assumed by experimental data on CE of long periodical variations. Differed from radiowaves, $\mathrm{CE}$ of low-frequential variations reveals an essential dependence on the primary field polarization. By the way of polarization of a falling electromagnetic field as to the coast line one may distinguish two types of $\mathrm{CE}$ in long periodical variations. If in a local, rectiangular system of coordinates $(X, Y, Z)$ the axis $X$ is directed along the coast-line, then:

CE-1. The magnetic vector of a falling field $H_{0} e^{i \omega t}=H_{0}\left(0, H_{0 y}, 0\right)$ ( $\omega$-circle friquency, $t$-time) is directed perpendicularly to the coast line. In this case electric currents run along the coast-line, that is $E=E_{x}$. From Maxwell's equations it follows that CE- 1 is first of all expressed in an anomalous behaviour of a normal (to the coast line) horizontal component of the magnetic field $H_{y} \sim \frac{1}{\omega} \frac{\partial E_{x}}{\partial z}$ and in appearance of anomalies in the variations of the vertical component of the magnetic field $H_{z} \sim \frac{1}{\omega} \frac{\partial E_{x}}{\partial y}$ and $H_{x}=E_{y}=E_{z}=0$.

CE-2. The vector of a falling field $H_{0}\left(H_{0 x}, 0,0\right)$ is directed parallel to the coast line. In this case $\mathrm{CE}-2$ is expressed in an anomalous behaviour of an electric field. Currents run perpendicularly to the coast-line, that is $E_{y} \sim \frac{1}{\sigma} \frac{\partial H_{x}}{\partial z}$ ( $\sigma$-the medium conductivity), near the coast-line the vertical component of the electric field $E_{z} \sim \frac{1}{\sigma} \frac{\partial H_{x}}{\partial y}$ appears (unfortunately extremely few experimental data on $E_{z}$-registration are available [2]). And $E_{x}=H_{y}=H_{z}=0$.

The phenomena, observed in nature are, certainly, more complicated and richer, than the above-given scheme. To a certain extent the both effects are always met simultaneously. Nevertheless in general features the peculiarities of the both types of $\mathrm{CE}$ are experimentally observed at the stations Mirny (Antarktida) and Alushta (the coast of the Black Sea).

The information about the conditions, methology and results of electromagnetic measurements in Mirny are given in [3-7]. The distribution of ionospheric sources over the Eastern Antarctica is probably favourable for CE-1 appearance. A tangential (to the coast-line) component of the alternative magnetic field $H_{x}$ does not experience notable variations along the profile, perpendicular to the coast, but the normal $H_{y}$ and the vertical $H_{z}$ with $T \sim 1-120$ min. show a typical dependence, given in Fig. 1a, where the distances along the profile in $\mathrm{km}$. are put along the axis of abscissae, relative amplitudes of variations-along the axis of ordinates, and for a unit one assumes variations, undistorted by a coast-effect 
that is in the points, removed from the coast-line. The measurements of currents in sea showed that they run parallel to the coast-line. A relative amplitude of $E_{x}$-variation with 20-60 min. periods on the profile perpendicular to the coast-line is given in Fig. 1b. If we take two points at $10 \mathrm{~km}$ and $100 \mathrm{~m}$ from the coast, then the calculations show, that with approaching the coast due to the decrease of the sea depth, the value $E_{x}$ could about 3 times increase. As the observations have given 6-7 time increase, it follows that for CE a twotime increase of $E_{x}$ remains. The comparison of simultaneous records of $E_{x}$ and $H_{z}$ in sea shows a good similarity, but their relation does not remain stable for different periods but becomes less with a variation period increase. The relations $\frac{H_{z}}{H}=\left|\frac{H_{z}}{\sqrt{H_{x}^{2}+H_{y}^{2}}}\right|$ measured on the land near the coast, depending on the periods, are given in Fig. 2. Besides, Fig. 2

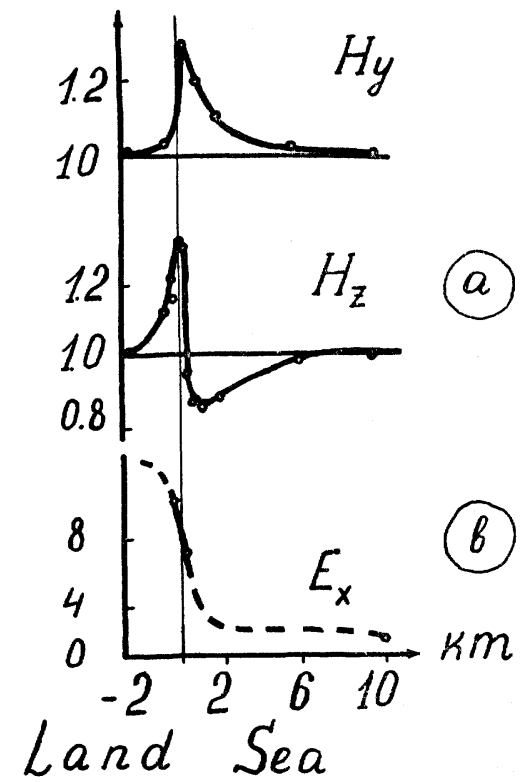

Fig. 1 .

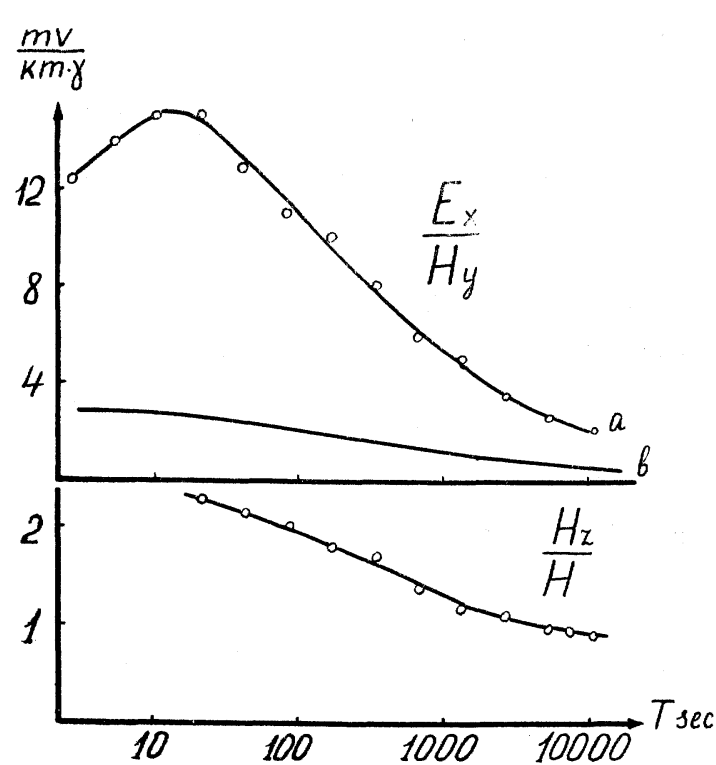

Fig. 2.

gives the values of an impedance $\left|\frac{E_{x}}{H_{y}}\right|$, defined from the observations for the point, situating at sea at $100 \mathrm{~m}$ distance from the coast (curve a), and a calculated (curve b) $\left|\frac{E_{x}}{H_{y}}\right|$ for a two-layer medium (sea-bottom) without the coast-effect taken into account. The exceeding of an experimental curve over a calculated one is explained by the presence of CE. CE-II (electrical vector is perpendicular to the coast) was observed in Alushta on the Black Sea [8]. The measurements of earth-currents along the profile, perpendicular to the coast-line, showed, that approaching the coast from the land side to $2-3 \mathrm{~km}$ distance from it $E_{y}$ increases at least by 3-4 times. This dependence is obtained for a broad interval of periods from 15 seconds up to several hours. The measurements of the earth-current field along a coast, cut up a little, showed that $E$ is approximately perpendicular to the coast-line everywhere. Some declinations from the normal are directed towards great deapths $(\sim 2000 \mathrm{~m})$, that follow the coastal shallow several kilometrs width. 
Thus, teoretical considerations and the results of the experiments allow to note the following main properties of $\mathrm{CE}$ :

Locality, which is the consequence of the locality of the gradients of low-semispace conductivity. CE intensity is directly connected with the value of impedance gradients, coast constitution and the bottom relief. $\mathrm{CE}$ dispersion is not equal for different components of the field.

\section{References}

E.L. Feinberg. Radiowave propagation along the Earth's surface. The A.S. of USSR, Moscow, 1960.

P.A. Vinogradov. Measurements of a vertical component of electrotelluric field in the lake "Baikal". The News of the A.S. USSR, series geophys. N1 1956, 83-86.

P.K. Senko. Unusual locality of magnetic variations in the region of Mirny. The Inf. Bulletin of the Soviet antarctic expedition N1, 1958, 81-82.

S.M. Mansurov. The causes of the locality of geomagnetic variations in the region of Mirny. The Inf. Bulletin of the Sov. antarctic exp. N2, 1958, 37-41.

P.K. Senko. The coast-effect in magnetic variations. The Inf. Bulletin of the Sov. antarctic exp. N4, 1959, 61-65.

L.N. Baranski, L. Naumenkov. Observations for the Earth's currents at the stations Mirny and Oazis in 1957. The Inf. Bulletin of the Sov. antarctic exp. N14, 1960, 74-78.

S.M. Mansurov. Some peculiarities of the alternative geomagnetic field in the region of the south-polar observatory Mirny. Summary "Magneto-ionospheric disturbances”. The A. of S. USSR, Moscow 1959, 64-66.

I.I. Rokitjansky. On the application of the magneto-telluric method on the anisotropic and non-uniform medium. The news of the A.S. USSR, series geoph. N11, 1961, 1607-1617. 